\title{
POLÍTICAS DE "FOMENTO" EN UNA SOCIEDAD EN TRANSICIÓN: DESAFÍOS Y OBSTÁCULOS. CHILE EN LA PRIMERA MITAD DEL SIGLO XX*
}

\author{
DEVELOPMENT POLICIES IN A TRANSITIONING SOCIETY: \\ CHALLENGES AND OBSTACLES. CHILE DURING THE FIRST \\ HALF OF THE 20 ${ }^{\text {TH }}$ CENTURY
}

\section{LUIS ORTEGA**}

\section{RESUMEN}

A partir de los críticos años de mediados de la década de 1920 y por prácticamente las siguientes cinco décadas, el Estado jugó un rol central en la conducción de los procesos económicos y sociales del país. En varias oportunidades en torno a su estructura central fue creada una suerte de "sub-estructura" de organizaciones asociadas en la forma de institutos de fomento y cajas de crédito, con diversos grados de autonomía y se legisló de manera copiosa -no siempre democráticamente- con una doble meta: adecuar la estructura del sector público para una gestión más eficiente con el objetivo central de "fomentar" el desarrollo productivo a través del diseño y aplicación de políticas ad hoc.

Palabras clave: Reorganización, fomento, estructura, sectores.

\section{ABSTRACT}

From the critical years in the mid-1920s, and for the next five decades, the state played a crucial role in shaping the economic and social processes in Chile. The central structure of the public sector, was reformed on several occasions, while regulatory bodies were created. Then, from 1926 onwards, a sort of "sub-structure" of agencies designed to boost production and support specific projects, was set up. At the same time, a copious

* Este artículo es fruto de los proyectos de 031164AY de la Dirección e Investigaciones Científicas y Tecnológicas (DICYT) de la Universidad de Santiago de Chile "La Modernización del Estado entre los dos gobiernos de Ibáñez: Concepto, Estructura y Funciones; 1924-1958”, y 1095107 del Fondo Nacional de Investigaciones Científicas y Tecnológicas (FONDECYT-Chile), "Contextos, actores y espacios para una redefinición del Norte Chico, 1925-1975. Una propuesta de análisis regional”.

** Ph.D. en Historia. Departamento de Historia, Universidad de Santiago de Chile. Santiago. Correo electrónico: luis.ortega.m@usach.cl 
legislation was enacted aimed at two objectives: first, to improve the performance of the public sector; second, to create adequate conditions for economic growth through the implementation of specifically designed public policies.

Keywords: Reorganization, development, structure, sectors.

Recibido: 19.06.12. Aceptado: 07.02.13.

\section{LOS DESAFÍOS DE LA DÉCADA DE 1920}

T

erminada la Primera Guerra Mundial (IGM), los gobernantes y líderes del mundo del capital vieron con perplejidad cómo su ilusión de que, terminado el conflicto, en cuestión de pocos años se restauraría el orden económico internacional vigente hasta 1912. Ello no solamente no se concretó, sino que el fin del conflicto abrió una era de inestabilidad internacional que se prolongó hasta más allá del fin de la Segunda Guerra Mundial. En ese contexto de incertidumbre, en el mundo que hasta agosto de 1914 había funcionado en el marco económico y comercial del librecambismo, y en particular en los países latinoamericanos que habían alcanzado un mayor desarrollo productivo en base a sus exportaciones, se comenzaron a verificar complejos y prolongados procesos de reforma del Estado, de cambios en la política económica y la implementación de políticas sociales (Albert 1988, I-II; Bulmer-Thomas, 1994, VI-VII-VIII-IX; Ellsworth, 1945, I-II).

La IGM constituyó un golpe devastador, pues no sólo dislocó su comercio exterior y su acceso a los mercados internacionales de las finanzas, los bienes de consumo y las tecnologías -los soportes materiales de todo el diseño político-institucional cuidadosamente articulado desde 1830-, sino que también puso en jaque el sistema político en la medida en que su crisis se agudizó y contribuyó a la profundización del conflicto social que desde el cambio de siglo se expresaba en una creciente conflictividad urbana, resultado esta de agudos problemas, entre los que destacaron el laboral y aquél referido a la calidad de vida en sus más variadas expresiones, entre las que resaltaron el de la alimentación, el laboral, el de la salubridad pública y el de la vivienda, por nombrar algunos (Deshazo, 1983, I-II-III). Es la época de la llamada "cuestión social", en otras palabras, de la irrupción en el país de fenómenos propios de las sociedades industriales, que desde el último cuarto del siglo XIX se incorporaban al elenco social, en un marco institucional cuyo diseño no consultaba un sistema político capaz de incluir a nuevos actores sociales y recoger, procesar y dar respuesta a todo tipo de 
demandas, entre las que no estuvieron ausentes las programáticas y las de representación política.

Gráfico I. Producto Interno Bruto, 1910-1938, en miles de millones de pesos de 1995.

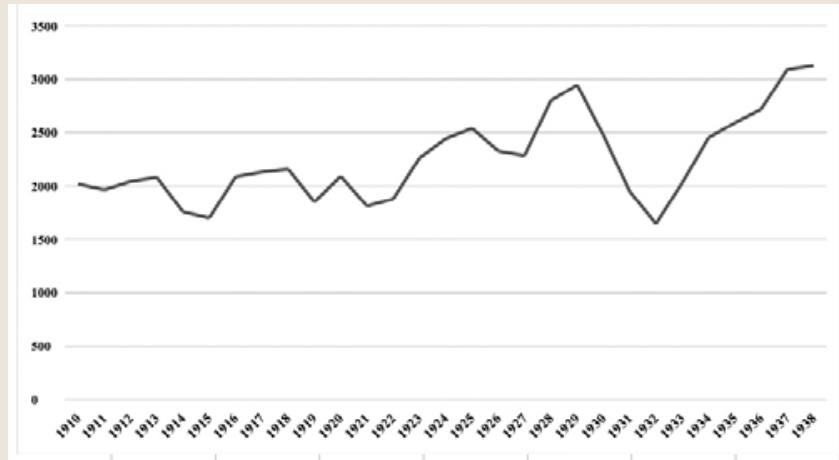

Fuente: Braun et al. (2000, pp. 22-23).

Los problemas económicos y sociales del período se agudizaron durante la primera mitad de la década de 1920. Para la economía nacional el fin de la IGM se tradujo en una fuerte caída en la demanda internacional por salitre, lo que marcó el comienzo del fin de la industria salitrera del Norte Grande. El impacto sobre el conjunto de la economía nacional fue inmediato y dramático: en 1919 el volumen de las exportaciones de nitrato fue sólo un cuarto de aquel 1918, y un quinto en valor.

Gráfico II. Producción anual de salitre, 1910-1934, en miles de toneladas métricas.

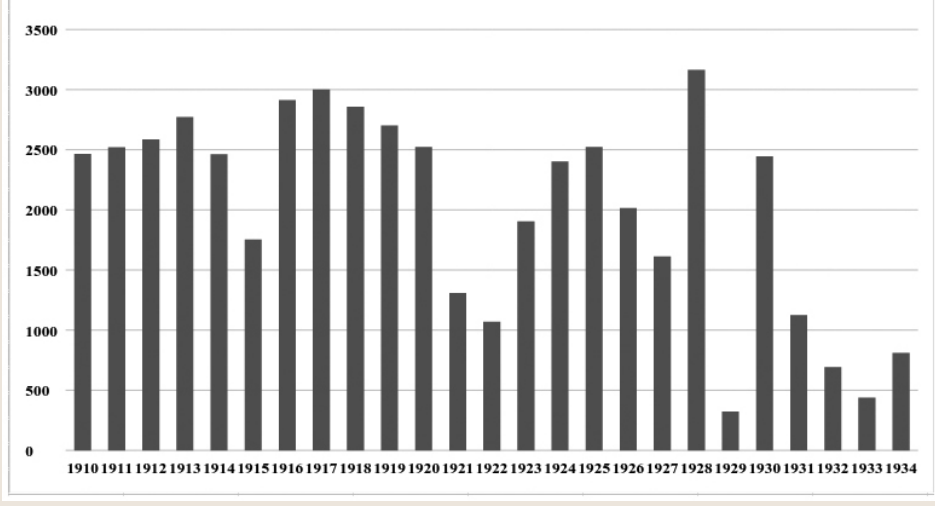

Fuente: Braun et al. (2000, p. 43). 
Lo que siguió fue, como ya se ha establecido, un prolongado período de inestabilidad que culminó dramáticamente al final de la década. Según los cálculos de José G. Palma (1984), “... la inestabilidad de la década de 1920 fue mayor que aquella de los cuatro años de la IGM y alimentó las posturas que desde comienzos de siglo demandaban la superación del 'modelo"' exportador y la instauración de una política económica, y las correspondientes instituciones, "que pusieran el acento en actividades productivas orientadas hacia el mercado interno, especialmente en manufacturas".

Sin embargo, las explicaciones económicas no bastan para dar cuenta a cabalidad de un fenómeno tan complejo. Es necesario para ello considerar un universo más amplio de variables, que en el caso de Chile en la década de 1920 demandan de la consideración de por lo menos cuatro: a) la creciente inestabilidad del sector externo; b) la virtual quiebra del Estado en 1922, c) la agudización de la conflictividad laboral a partir de 1919, y d) junto con ello, pero en una dimensión mayor, la agudización de los problemas sociales.

Es desde esa perspectiva que deben ser evaluados hoy los resultados de las "políticas de fomento productivo" que se implementaron en el país desde mediados de la década de 1920 hasta comienzos de la de 1960, sobre todo porque ellas fueron entendidas como una de las claves para lograr el crecimiento y el desarrollo. De acuerdo con el estado del conocimiento sobre el tema no es del todo osado preguntarse si en Chile hubo una política de desarrollo entre 1925 y 1961. En términos generales, es plausible proponer que no hubo "una política", sino un conjunto de decisiones que se adoptaron para incentivar y desarrollar la producción, en particular la industrial, pero que no se articularon en torno a un proyecto global. La mayor parte de las iniciativas diseñadas e implementadas en aquel período no estuvieron acompañadas "de un propósito correlativo por precisar los fines de la política estatal; antes al contrario, ese esfuerzo llevó consigo casi siempre una cuota no despreciable de incapacidad para administrar con eficiencia la creciente maquinaria pública" (Molina, 1972, pp. 25-26), además de un importante componente de improvisación. Así enfocado el período es dable proponer que la contribución de las políticas públicas al desarrollo social y económico fue limitada por cuestiones de orden institucional, si bien permitieron considerables aumentos en la producción de bienes y servicios. Las limitaciones tuvieron origen en cuestiones de carácter institucional, en la medida en que si bien al Estado se le adosaron cada vez más funciones y tareas, no hubo un rediseño estratégico de éste; más bien se trató de adecuaciones que buscaron el logro de mayores grados de eficiencia en la gestión pública. 
El objetivo estratégico de los gobernantes fue "fomentar" la actividad productiva, pero sin por ello innovar en cuestiones cruciales, como lo son el tema de las reglas del juego y aquél de las tecnologías de productos y de procesos. En otras palabras, en la formulación de las políticas públicas estuvo ausente la concepción de "desarrollo", entendido este no tan sólo como el aumento de la producción y el incremento prolongado y constante de la renta nacional para el financiamiento de más crecimiento, sino como el movimiento ascendente de la totalidad del sistema social (Rostow, 1960; Myrdal, 1974). Esa concepción y práctica del manejo de la economía estuvo a la base de la breve duración de la primera etapa de las políticas de fomento.

\section{EL PAÍS EN LA ENCRUCIJADA}

Desde mediados de la década de 1920 y en la medida en que los shocks externos comenzaban a golpear con mayor intensidad al país, los debates acerca del rol del Estado así como la orientación que debía darse a la economía, iniciados en torno a la conmemoración del Centenario, se intensificaron. A los macroproblemas ya enumerados deben agregarse dos acontecimientos políticos fundamentales que agudizaron las tensiones: en primer lugar el bloqueo por parte de la mayoría en el Congreso Nacional a las iniciativas del gobierno reformista de Arturo Alessandri -instalado en 1920- y que buscaban atenuar y controlar los crecientes conflictos laborales e introducir una módica cuota de cobertura social en una sociedad con vergonzosos niveles de pobreza y destitución; en segundo lugar, las crecientes dificultades fiscales. El segundo factor fue la intervención militar de 1924 en el escenario político.

Fue en ese contexto que emergió con fuerza la propuesta de los sectores medios de la sociedad -profesionales civiles (abogados, ingenieros, médicos y algunos profesores) y militares- que en lo esencial planteaba dos cuestiones fundamentales cuyo objetivo estratégico era cambiar la orientación del sistema productivo. Lo central del programa o "ideología" de esos sectores proponía: 1) el desarrollo de lo que denominaron "la industria nacional", sin mayores especificaciones y 2) el intervencionismo del Estado en el campo económico. A un intervencionismo limitado a la adopción de medidas indirectas, fundamentalmente en el ámbito de la política comercial, se añadió un intervencionismo directo; las capas medias presionaron hasta lograr que el Estado asumiera la función de empresario en los secto-

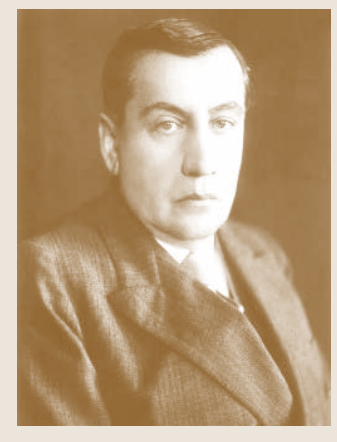

A. Alessandri Palma 
res insuficientemente atendidos por la iniciativa privada, evitando siempre entrar en competencia con ella (Carmagnani, 1984, III).

Gráfico III. Huelgas, 1907-1922.

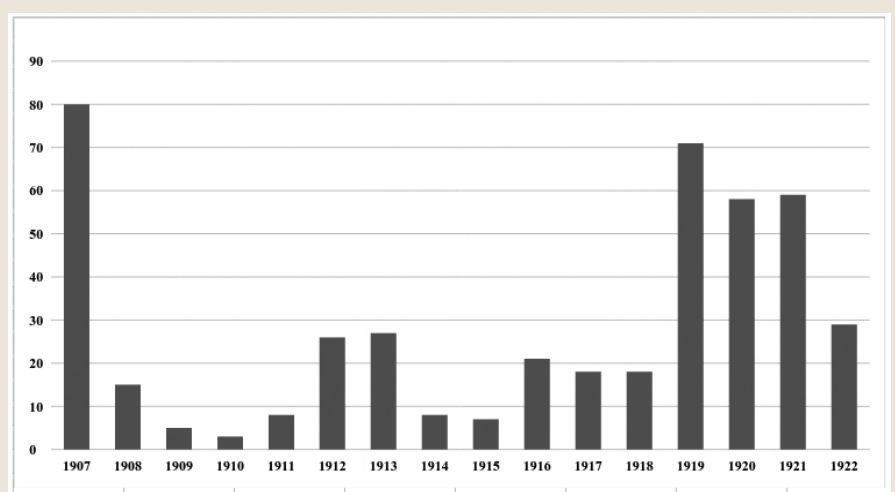

Fuente: DeShazo (1983, pp. 201-240).

Gráfico IV. Índice de la producción de manufacturas 1910-1939 (1960=100).

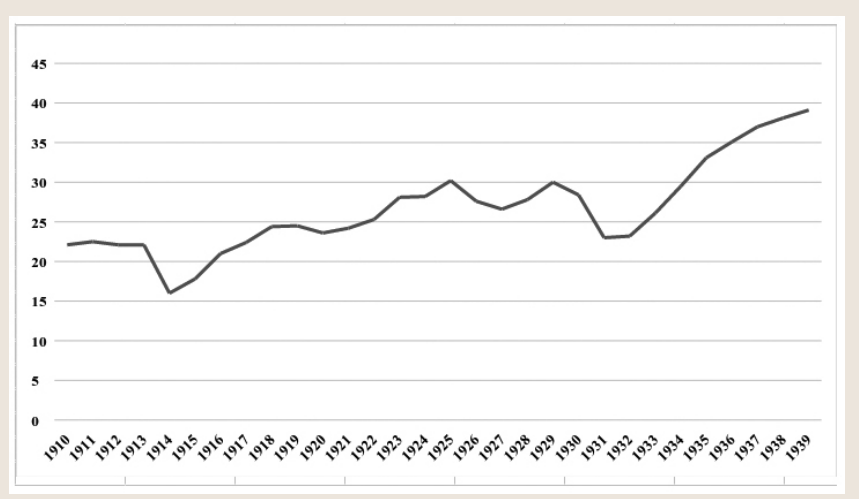

Fuente: Braun et al. (2000, p. 103).

El Presidente Arturo Alessandri no fue ajeno a ese debate, que se verificó con el trasfondo de una importante expansión de la producción fabril, tal cual se aprecia en el Gráfico IV, y en su último Mensaje al Congreso Pleno abogó por políticas de fomento de la producción industrial más decididas, a la vez que anunció que para esos efectos su interlocutor sería la Sociedad de Fomento Fabril (Mensaje 1924, pp. 63-67); en otras palabras, el empre- 
sariado, el que desde fines del siglo XIX había ejercido fuertes presiones sobre el gobierno y el Congreso Nacional para obtener tarifas de importación que protegieran las actividades de sus asociados.

Sin embargo, el Presidente Alessandri se quedó sin tiempo, pues a comienzos de septiembre de 1924, un actor hasta entonces silente, pero no por ello no deliberante -los militares-, decidieron intervenir el Estado. El Manifiesto militar, entregado al Presidente en los primeros días de septiembre de aquel año tenía un alto contenido de cuestiones laborales, productivas, educacionales y sociales que se comenzaron a plasmar en la implementación de diversas decisiones políticas que redundaron en la creación de nuevos organismos de regulación tanto del quehacer estatal como del proceso económico (Drake, 1978, I-II; Arias, 2011).

A partir de 1926, ya con los militares en control del Estado, comenzó la modificación del diseño de la estructura central de éste, en la que se destacó por una parte la reestructuración de los ministerios que comprendió la creación en 1927 del Ministerio de Fomento y, por otra, en el inicio de la creación de un "aparato para-estatal", constituido por un conjunto creciente de agencias e instituciones autónomas, cuyo objetivo estratégico era fomentar la actividad productiva en general y la industrial en particular (Ibáñez, 1983, pp. 45-49).

Paralelamente, en el plano político el coronel Carlos Ibáñez del Campo comenzó a acumular poder al punto de controlar el gobierno desde diversas carteras ministeriales y eventualmente en calidad de Vicepresidente de la República. En esta última condición, en el mes de mayo de 1927, el coronel presentó un "Manifiesto al país" en que explicitó su programa de gobierno $-\mathrm{y}$ de alguna manera un proyecto de nuevo ordenamiento del país-, en que de esta forma se consolidó la hegemonía de las ideas y posturas de los sectores medios en cuanto a desarrollo productivo y rol del Estado en la economía y la sociedad. Para Ibáñez, en la condición de candidato presidencial, uno de los ejes de su política económica sería el cimentar "en forma permanente, aun a costa de grandes sacrificios, la situación de nuestra hacienda pública, sobre la base de severa economía e intensivo fomento de la producción y las industrias nacionales". En otras dos dimensiones las nuevas perspectivas ocuparon un lugar central en el programa de gobierno; en cuanto a educación aseveró que "la juventud chilena reclama[ba] una atención especial de parte del gobierno en orden a proporcionarle una educación científica, física y moral que le capacite para la lucha por la vida y para las funciones productivas", con lo que formuló propuestas que redundaron ese año en la total reestructuración del sistema educacional, en cuyo

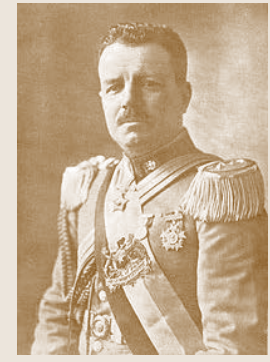

C. Ibáñez del C. 
contexto cabe destacar el proyecto de instauración de un sistema de Educación Técnico-Profesional (Ibáñez del Campo, 2001 [1927], pp. 156-158).

Un campo de acción adicional, en las restructuraciones y nuevos diseños organizacionales y administrativos iniciados en los años 20, fue el de la regionalización, entendida ésta como parte de la búsqueda de soluciones a los acuciantes problemas económicos que enfrentaba el país (Arias, 2011). En esta dimensión el propósito del gobierno fue efectuar una profunda reestructuración administrativa, cuyo objetivo estratégico no era sólo mejorar la eficiencia de la gestión pública en ese nivel, sino contribuir a través de ello "a la solución de los problemas nacionales que afectan a la agricultura y a las extensas y ricas regiones del norte y sur del país, cuyo progreso está aún detenido por el absorbente centralismo establecido hasta ahora como norma de gobierno y administración (Ibáñez del Campo, 1927, p. 157).

Pocos días después de publicado su manifiesto, Carlos Ibáñez hizo su balance del estado de la nación ante el Congreso Pleno, y en su discurso volvió sobre los temas incluidos en su programa desde la perspectiva de la reorganización orgánica ministerial que pocos meses más tarde derivó en la creación, por decreto, del Ministerio de Fomento conjuntamente con diversas cajas de crédito e institutos de cuya función sería estimular el desarrollo productivo, entre los que se destacaban el Instituto de Crédito Industrial, la Caja de Crédito Minero, la Caja de Crédito Agrario y la Caja de Crédito Carbonífero. El objetivo de estas readecuaciones era "llevar a cabo en forma sistemática los estudios necesarios para el fomento y protección de nuestras industrias", lo que pasaba a ser la "atención preferente del Estado... el progreso y desarrollo de la industria del país", para lo cual el gobierno haría "cualquier sacrificio para obtener nuestra independencia económica mediante la implantación de medidas que protejan la industria nacional" (Mensaje 1927, p. 16).

La trampa en que había caído Ibáñez era el de intentar "fomentar" la producción de un sistema económico que evidenciaba complejos arcaísmos y que para ser resueltos requerían de cambios estructurales profundos. Ese continuó siendo el dilema de Chile por las siguientes cuatro décadas.

\section{III. ¿EL NACIMIENTO DE UN MITO?}

En la misma perspectiva conceptual de las reformas al aparato estatal a partir de la segunda mitad de la década de 1920, en el año 1939 fue creada por 
ley la Corporación de Fomento de la Producción (CORFO), para dar impulso al conjunto del aparato productivo, y en particular al sector industrial. Esta entidad, con el transcurso de las décadas se transformaría, desde el punto de vista de la "sabiduría convencional", en $e l$ instrumento estatal fundamental para alcanzar el desarrollo y la diversificación productiva (Ortega et al., 1989). Es cierto que la CORFO fue, hasta mediados de la década de 1950, vital para el desarrollo de grandes proyectos -la siderurgia, el desarrollo de algunos sectores de la industria metalúrgica, la remolacha, la celulosa y el papel, el plan nacional eléctrico y la producción de petróleo-, pero también lo es que, a mediados de aquella década, durante el segundo gobierno de Ibáñez, esos grandes proyectos se agotaron junto a la "ilusión del desarrollo". Entonces el país entró en un ciclo de fuerte inestabilidad económica -alta inflación y bajo crecimiento-, radicalización social y política que culminó en 1973.

Los análisis económicos acerca del desempeño de la economía chilena en general y de su sector industrial, en particular, han llegado a un balance casi definitivo acerca del crecimiento y del grado de desarrollo logrado durante el período a que se refiere este estudio (Muñoz, 1995, pp. 1-27).

La CORFO y todas las instituciones públicas de fomento desarrollaron sus actividades sin un "plan maestro", con limitados recursos humanos calificados y con desconocimiento de la dotación real de recursos naturales del país, así como de las cuentas nacionales; no en vano, el mandato que CORFO recibió en 1939, de generar un "Plan Nacional de Desarrollo", sólo se plasmó en 1961. Hasta entonces, en todos los ámbitos de la producción, la institución desarrolló iniciativas puntuales, como lo fueron en sus primeros años los célebres Planes de acción inmediata de 1939 y luego proyectos específicos, algunos de ellos de indudable trascendencia, como el de la siderurgia, en asociación con el sector privado (Muñoz, 1986). En lo que se refiere al plano productivo en general, la CORFO y las demás instituciones públicas descentralizadas debieron abocarse no sólo a implementar proyectos productivos innovadores, sino también a atender las demandas de diversos sectores productivos "tradicionales", tanto en la agricultura como en la minería, con lo cual contribuyeron a su reproducción y reforzamiento $\mathrm{y}$, en muchos casos, a la persistencia de sus prácticas productivas tradicionales, arcaicas. Las políticas públicas destinadas al fomento productivo estuvieron, por lo tanto, signadas por la improvisación y por importantes contradicciones. 
Gráfico V. Producto Interno Bruto, 1940-1958, en miles de millones de pesos de 1995.

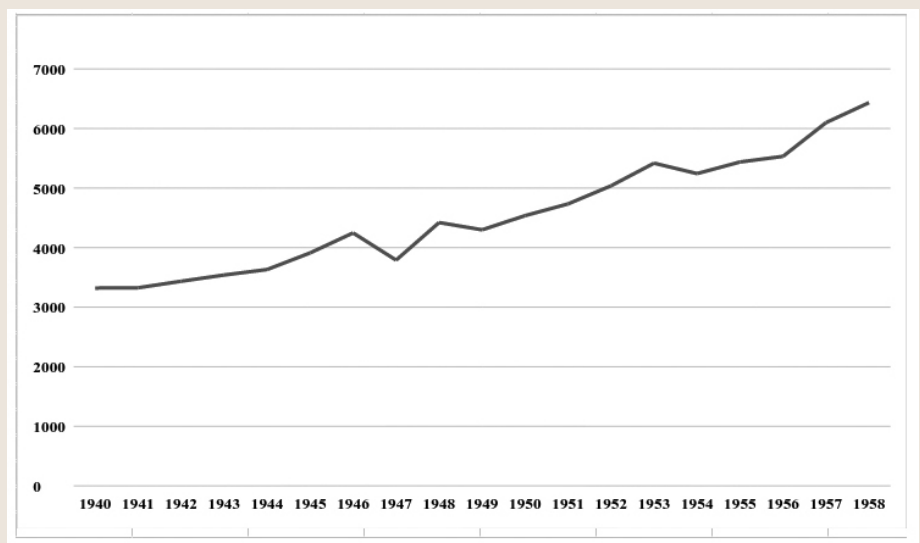

Fuente: Braun et al. (2000).

Gráfico VI. Tasa anual de inflación 1938-1958.

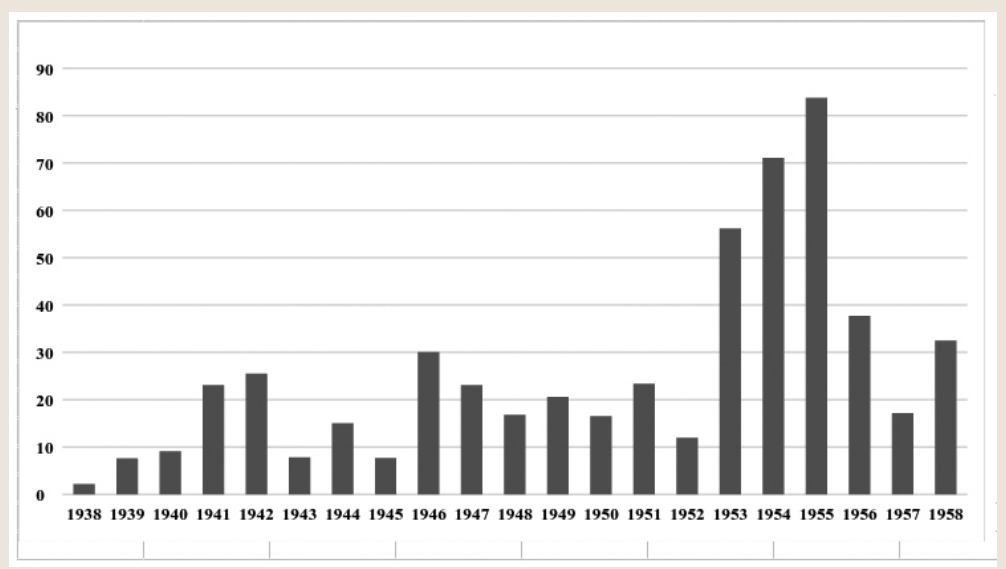

Fuente: Braun et al. (2000, p. 100).

Si en Chile la aspiración de hacer del país uno con una importante base industrial se frustró, ello fue, en importante medida, el resultado de que el Estado y la CORFO debieron distraer sus capacidades y limitados recursos para asistir a sectores productivos inmersos en profundas crisis, lo cual les restó capacidad de acción para diseñar objetivos específicos de desarrollo productivo e investigación en tecnologías de productos y procesos. Ese in- 
volucramiento restó efectividad a las acciones de las diversas agencias estatales, en la medida en que debieron destinar un importante volumen de recursos financieros y humanos a sustentar actividades productivas cuya viabilidad era, por lo menos, discutible. Un ejemplo de ello es lo que aconteció en el ámbito de la minería.

En agosto de 1939 la CORFO publicó su Plan de acción inmediata para la minería que contenía un conjunto de disposiciones, según el documento, "realizables mediante préstamos a particulares o a la Caja de Crédito Minero, o por asociaciones de la Corporación con otras personas" (CORFO, 1939, p. 3). Más importante aún fue el que las "Recomendaciones" del Plan estuvieran constituidas por un amplio abanico de acciones de compleja realización, dadas la demandas organizativas, financieras y técnicas que ellas implicaban, entre las que cabe destacar "fondos necesarios para resolver definitivamente el suministro de energía eléctrica" de las áreas de Copiapó y El Salado, "un plan de construcción de caminos de acceso a los nuevos centros productores de minerales; otro de mejoramiento de los más importantes caminos troncales de Atacama y un acuerdo para la conservación conjunta de los caminos netamente mineros". Junto con ello la CORFO recomendaba a la Empresa de Ferrocarriles del Estado "la construcción de un tipo determinado de carros para el transporte simultáneo a granel en la Red Norte, de lotes de minerales de 5 y 2,5 toneladas" y, finalmente, comprometía sus esfuerzos para asistir a la Caja de Crédito Minero en la elaboración de un "proyecto de construcción de campamentos obreros en todas las plantas de la Caja, incluyendo edificios adecuados para escuelas en aquellas que aún no poseen, de acuerdo con la Caja de Habitación Popular" (CORFO, 1939, p. 4).

Tan ambicioso y demandante Plan no fue sólo el fruto del voluntarismo de los ingenieros de la CORFO. A pesar del importante grado de autonomía de que gozó la entidad, por lo menos hasta 1970, las presiones y demandas sectoriales que debió enfrentar desde diversos ámbitos -entre los que también se contaban el gobierno central y el Congreso Nacional- le obligaron a generar respuestas a múltiples demandas no siempre viables, con lo cual, sin duda, se vio deteriorada la efectividad de su acción. En este caso, tal como lo deja explícito el mismo documento, el Plan fue formulado "en conformidad con los acuerdos del Congreso Minero de Copiapó de 1937" y, tal vez lo más importante, "en concordancia con las ideas expresadas en el Plan de Fomento de la Comisión nombrada por la Caja de Crédito Minero". Para entonces las críticas de que era objeto la Caja eran profundas y extendidas, pues se registraban en las provincias mineras, en la prensa local y en ambos hemiciclos del Congreso desde el año 1930. 
Los acuerdos del Congreso Minero de Copiapó constituyeron una sistematización y reafirmación de las peticiones que desde la década de 1930 pasaron a constituir demandas, que el sector en crisis comenzó a formular desde la última década del siglo XIX, y que, según Alberto Herrmann (1900, p. 101), convirtieron a una actividad otrora autónoma en peticionaria del Estado. Las demandas emanadas de ese evento fueron amplias: a) Creación de un fondo de regulación de la política monetaria y de crédito con las modalidades adecuadas a la distribución de divisas con un cambio especial para la minería; b) La instalación de una Fundición Nacional y de una refinería electrolítica y la dictación de una ley que faculte al Presidente de la República para expropiar los yacimientos de fundentes que los organismos del Estado consideren necesarios para el normal establecimiento de la fundición; c) La creación del Ministerio de Minas; propender a la nacionalización de la minería, a la explotación de las minas paralizadas por medio de un avío obligatorio e implementación de una legislación social que dé unidad a los servicios de Previsión, orientar las actividades de la Caja de Seguro Obligatorio, en el sentido de propender en forma efectiva a mejorar y levantar el nivel oral y cultural de los obreros; que se realice el proyecto ya aprobado de construcción del Hospital Regional para tuberculosos; d) Fondos necesarios para desarrollar un plan de caminos, en particular de acceso o caminos transversales al FF.CC. Dotación de agua a las zonas más azotadas por la falta de ese elemento. Habilitación de las aguadas existentes y estudio de las napas a fin de utilizarlas y d) Reforma de las disposiciones tributarias a que estaba sometido el sector (Anónimo, 1937, pp. 1591-1613).

Esas demandas tenían una larga trayectoria, pues comenzaron a ser formuladas en los últimos años del siglo XIX y en el primer cuarto del XX, y adquirieron una presencia permanente, tanto en la prensa local como en el Congreso Nacional, en particular en la Cámara de Diputados. En 1895 el diputado José Rafael Balmaceda Fernández -Liberal Democrático- manifestó que "llegue el nuevo año sin que los legisladores inclinen por primera vez la mano protectora del Estado sobre aquella provincia, que con la de Copiapó impulsaron poderosamente en otra época el progreso, la riqueza y el bienestar nacional" (CD SO 2 , 1895, p. 35). En 1897 algunos diputados solicitaron "garantía del Estado, por el término de 20 años, para el interés del cinco por ciento anual de un capital de dos millones quinientos mil

\footnotetext{
${ }^{2}$ Cámara de Diputados Sesión Ordinaria.
} 
libras esterlinas que se propone invertir en implantar i explorar en el país establecimiento de beneficio de minerales, i en la explotación de minas, ya sea por cuenta propia o en aviación" (CD SE ${ }^{3} 1897-1898$; 9a 29 X 1897; p. 152. CD SO 1898, 57a 7 IX 1898, pp. 1088-1089; CD SO, 1913, 16a , 28 VI 1913; CD SE, 1916, 58ª 18 VII 1916; 1917-1918, 19a , 32a ; 3 XII 1917, 18 VII 1918, 553 y 963$)$.

En medio de la IGM, y en la medida en que se agudizaron los problemas de la minería tradicional, comenzaron a plantearse proyectos que incluían la "protección a la industria minera" a través de la compra de "minerales y pastas de cobre" y el establecimiento de fundiciones por parte del Estado (CD SE, 1916, Sesión 58a, 18 VII 1916; 1917-1918, sesiones $19^{\mathrm{a}}$ y $32^{\mathrm{a}}$ en 3.XII.1917 y 18 VII 1918, pp. 553 y 963, respectivamente. El Atacameño, El Amigo del País, 1928 y 1929). En las más extremas condiciones de la primera mitad de la década de 1930, esas demandas fueron centrales en las conclusiones de por lo menos dos congresos mineros.

La respuesta pública se tradujo en que hasta los últimos años de la década de 1950 la CORFO y la Caja de Crédito Minero (CACREMI) se involucraron en una compleja trama de demandas contradictorias y de difícil resolución, pues las peticiones de los actores de las provincias mineras tradicionales no sólo eran variadas en cuanto a contenido, también lo eran de acuerdo a quienes las planteaban, ya fuesen los medianos o los pequeños productores, quienes de las demandas enumeradas pasaron a cuestiones de carácter operacional como lo fueron la provisión de agua, herramientas, provisiones, viviendas, servicios sociales y asistencia sanitaria y técnica para una masa de operarios independientes, quienes vendían el producto de su trabajo diario a más de 40 agencias de compra del gobierno a un precio fijado levemente por debajo de la cotización internacional. De esa manera, la pequeña y mediana minerías se transformaron en "un sistema gigante de pirquén, con el gobierno [y sus agencias] como habilitador" (Pederson, 2008, p. 293).

Sin embargo, a poco andar -a mediados de la década de 1930- tanto las actividades de la CACREMI, así como las desarrolladas por la CORFO en el ámbito minero, a través de aquella entidad o por iniciativa propia, comenzaron a ser objeto de ácidas críticas en las provincias mineras del norte en donde se acusó que la CACREMI favorecía los intereses de los medianos mineros -aquellos que contaban con plantas de procesamiento de minerales propias- e incluso a intereses extranjeros, en desmedro de los

\footnotetext{
${ }^{3}$ Cámara de Diputados Sesión Extraordinaria.
} 
“pequeños mineros". En una primera instancia fueron las asociaciones locales las que recogieron las crecientes protestas, pero en corto tiempo ellas llegaron al Congreso Nacional. En ese ámbito, en la década de 1940 las denuncias comprendieron desde erradas decisiones, en cuanto a la adopción de tecnologías de procesamiento de los minerales, discrecionalidad en el enrolamiento de personal y, hacia el final de la década y comienzos de la de 1950, acerca de recurrentes casos de corrupción (CD SO, 11ª).

El conjunto de demandas que debió enfrentar la CORFO desde el comienzo de sus operaciones, como ya lo experimentaban los gobiernos con anterioridad al colapso de la economía en los primeros años de la década de 1930, fue múltiple y variado y no fue sólo el sector minero el único que lo planteó. La propia CORFO se comprometió en planes piloto, cuyo objetivo era introducir algún grado de modernización en diversos sectores productivos en el agro, como lo fueron el "Plan Chillán”, el "Plan Punitaqui” y el plan forestal, base años más tarde para el desarrollo de la industria productora del papel y la celulosa. De esa manera, paradojalmente, desde fines de la década de 1920 las instituciones públicas contribuyeron a ahondar la contradicción más duradera del país, aquella entre tradición y modernización. Durante 20 años ese fue el producto, no deseado, de las políticas públicas de fomento, que luego se tornaron "desarrollistas" -crecientemente de inspiración cepaliana- y a mediados de la década de 1950 todo ello desembocó en "la crisis integral de Chile" y en "el desarrollo frustrado".

Si políticamente la creación de la CORFO fue compleja y demandó de un compromiso de largo plazo, según el cual el sector agropecuario no fue objeto de políticas transformadoras sino hasta la década de 1960, también en el seno del propio Estado hubo resistencias respecto de iniciativas que pretendían dar una mayor coherencia al intento de desarrollar la base industrial del país.

Entonces, también en este sentido es plausible formular una hipótesis provisional: el limitado éxito de las iniciativas industriales en particular y aquellas diseñadas para los demás ámbitos productivos hacia mediados de la década de 1950 fue, en parte importante, el resultado de los problemas sistémicos al interior del actor fundamental del período: el Estado. Tanto por incapacidad derivada de la improvisación, de sus falencias organizativas y los limitados recursos humanos calificados y de fuertes intereses contradictorios en su seno, el ente público no estuvo en condiciones de diseñar e implementar una política pública propiamente tal; en palabras de Jorge Ahumada, había "una crisis de organización en el sentido de que las formas 
preponderantes de organización son incapaces de realizar con un grado razonable de eficiencia las tareas que cada uno debe realizar. La organización administrativa del estado es ineficiente; la organización política del estado es ineficiente... [también] la del sistema educativo" (Ahumada, 1966, pp. 29-30). Cuando la CORFO estuvo en condiciones de cumplir su mandato, en 1961, ya era tal vez demasiado tarde, pues desde mediados de la década de 1950 el país había entrado en una crisis de largo aliento.

\section{CONCLUSIÓN... PROVISIONAL}

Las políticas públicas implementadas en Chile a partir de la segunda mitad de la década de 1920 tuvieron un contenido ideológico en que se plasmó la aspiración a la modernización y el desarrollo en una compleja combinación con el fomento a las actividades productivas tradicionales.

El resultado de ello fue un importante grado de modernización productiva de algunos sectores que permitió que el producto interno bruto creciera a una modesta tasa acumulativa anual de 2,6 por ciento entre 1925 y 1955; en el mismo período, la producción industrial creció al 5,6 por ciento anual mientras que la agricultura y la minería crecieron al 1,9 y 0,1 por ciento, respectivamente. En otras palabras, el esfuerzo modernizador por el lado de la industrialización sacó al país de su retardo en ese ámbito, pero no "arrastró" a la modernización a los sectores más tradicionales, y a mediados de la década de 1950 se estancó. Simultáneamente, en 1955 la tasa de inflación anual se convirtió en un "verdadero flagelo" y alcanzó un inédito 85,5 por ciento, en gran medida como resultado del gasto fiscal basado en endeudamiento -interno y externo- que desde la década de 1920 financió los planes de desarrollo productivo (Hirschman, 1964, pp. 182-254).

Entre 1925 y 1961 las políticas de fomento no enfrentaron los problemas más fundamentales del país en el ámbito económico, sino que sólo permitieron algunos importantes avances productivos, en particular en el sector industrial. Pero también esas políticas contribuyeron a mantener la vigencia de arcaísmos productivos y laborales a un alto costo, y ello contribuyó de manera decisiva a generar la "crisis integral" de la década de 1950, y crearon la antesala de la etapa de las reformas estructurales iniciadas en 1964 y de la crisis general de la década de 1970. 


\section{REFERENCIAS}

Albert, B. (1988). Latin America and the First World War. The Impact of the War on Brazil, Argentina, Peru and Chile. Cambridge: Cambridge University Press.

Ahumada, J. (1966). En vez de la miseria. Santiago: Universitaria.

Anónimo (1937). "Conclusiones del Congreso Minero de Copiapó". Boletín Minero de la Sociedad Nacional de Minería, año LIII, vol. XLIX.

Arias, K. (2011). "Ideas, intentos y fracasos en el proceso modernización administrativa entre las dos administraciones de Ibáñez (1927-1958)”. Ponencia inédita presentada a las XIX Jornadas de Historia de Chile, Santiago.

Braun, J.; Braun, M.; Briones, I. (2000). Economía chilena 1810-1995. Estadísticas históricas. Santiago: Instituto de Economía, P. Universidad Católica de Chile.

Bulmer-Thomas, V. (1994). The Economic History of Latin America Since Independence. Cambridge: Cambridge University Press.

Carmagnani, M. (1984). Estado y sociedad en América Latina 1850-1930. Barcelona: Crítica.

CORFO (Corporación de Fomento de la Producción) (1939). Plan de acción inmediata para la minería. Santiago: CORFO.

DeShazo, P. (1983). Urban Workers and Labor Unions in Chile, 1902-1927. Madison: University of Wisconsin Press.

Drake, P. W. (1978). Socialism and Populism in Chile, 1932-1952. Urbana: University of Illinois Press.

Ellsworth, P. T. (1945). Chile. An Economy in Transition. New York: Macmillan.

Herrmann, A. (1900). "Estado de la minería del cobre en Chile”. Boletín de la Sociedad Nacional de Minería, 40, 30.IV.

Hirschman, A. O. (1964). "Inflación en Chile". Estudios sobre política económica en América Latina (En ruta hacia el progreso). Madrid: Aguilar.

Ibáñez, A. (1983). "Los ingenieros, el Estado y la política en Chile: del Ministerio de Fomento a la Corporación de Fomento: 1927-1939". Historia, 18.

Ibáñez del Campo, C. (2001). "Manifiesto al país de candidato a la Presidencia de la República don Carlos Ibáñez, 12.V.1927)”. En Sofía Correa et al., Documentos del siglo XX chileno. Santiago: Sudamericana.

Molina Silva, S. (1972). El proceso de cambio en Chile. La experiencia chilena 1965-1970. Santiago: Ilpes.

Muñoz, O. (1986). Chile y su industrialización: pasado, crisis y opciones. Santiago: CIEPLAN.

- (1995). Los inesperados caminos de la modernización económica. Santiago: Universidad de Santiago de Chile.

Myrdal (1974). "What is Development?". Journal of Economic Issues, vol. VIII, No 4.

Ortega, L. et al. (1989). Corporación de Fomento de la Producción. Cincuenta años de realizaciones. Santiago: Universidad de Santiago de Chile. 
Palma, J. G. (1984). “Chile 1914-1935: de economía exportadora a sustitutiva de importaciones”. En Colección de Estudios Cieplan, No 12, 61-88.

Pederson, L. (2008). La industria minería del Norte Chico. Chile. Santiago: RIL.

Rostow, W. W. (1960). The Stages of Economic Growth: A non-communist manifesto. London \& New York, Cambridge University Press. 